\title{
An app-, web- and social support-based weight loss intervention for adults with obesity: the 'HelpMeDolt!' feasibility randomised controlled trial
}

Sharon Anne Simpson ${ }^{1 *}$ D, Lynsay Matthews ${ }^{1}$, Juliana Pugmire ${ }^{1}$, Alex McConnachie ${ }^{2}$, Emma Mclntosh³, Elinor Coulman ${ }^{4}$, Kathryn Hughes ${ }^{5}$, Mark Kelson6, Sarah Morgan-Trimmer, Simon Murphy,

Olga Utkina-Macaskill ${ }^{1}$ and Laurence Anthony Russell Moore ${ }^{1}$

\begin{abstract}
Background: Social support has an important role in successful weight loss. The aim of this study was to assess the feasibility and acceptability of an app-, web- and social support-based intervention in supporting adults with obesity to achieve weight loss.

Methods: The intervention and evaluation methods were tested in a feasibility randomised controlled trial. Adults in the Greater Glasgow and Clyde Health Board area of Scotland with a body mass index $\geq 30 \mathrm{~kg} / \mathrm{m}^{2}$ were recruited and randomised 2:1 (intervention to control). The feasibility and acceptability of the intervention and trial methods were assessed against pre-specified progression criteria, via process, economic and outcome evaluation. Three primary outcomes were explored: BMI, diet and physical activity, as well as a number of secondary outcomes. The intervention group had access to the HelpMeDolt! intervention for 12 months. This encouraged them to (i) set goals, (ii) monitor progress and (iii) harness social support by inviting 'helpers' from their existing social network. The control group received a healthy lifestyle leaflet.

Results: One hundred and nine participants were recruited, with 84 participants (77\%) followed-up at 12 months. The intervention and trial methods were feasible and acceptable. Participants and helpers were generally positive. Of the 54 (74\%) participants who downloaded the app, 48 (89\%) used it. Interview data indicated that HelpMeDolt! promoted social support from existing social networks to support weight loss. This support was often given outside of the app.

Outcomes were compared using linear regression models, with randomised group, the baseline measurement of the outcome, age and gender as predictor variables. These analyses were exploratory and underpowered to detect effects. However, all pre-specified primary outcome effects (BMI, diet and physical activity) had wide confidence intervals and were therefore consistent with clinically relevant benefits. Objective physical activity measures perhaps (Continued on next page)
\end{abstract}

\footnotetext{
* Correspondence: Sharon.Simpson@glasgow.ac.uk

${ }^{1} \mathrm{MRC} / \mathrm{CSO}$ Social and Public Health Sciences Unit, Institute of Health and

Wellbeing, University of Glasgow, Berkeley Square, 99 Berkeley Street,

Glasgow G3 7HR, UK

Full list of author information is available at the end of the article
}

(c) The Author(s). 2020 Open Access This article is licensed under a Creative Commons Attribution 4.0 International License, which permits use, sharing, adaptation, distribution and reproduction in any medium or format, as long as you give appropriate credit to the original author(s) and the source, provide a link to the Creative Commons licence, and indicate if changes were made. The images or other third party material in this article are included in the article's Creative Commons licence, unless indicated otherwise in a credit line to the material. If material is not included in the article's Creative Commons licence and your intended use is not permitted by statutory regulation or exceeds the permitted use, you will need to obtain permission directly from the copyright holder. To view a copy of this licence, visit http://creativecommons.org/licenses/by/4.0/. The Creative Commons Public Domain Dedication waiver (http://creativecommons.org/publicdomain/zero/1.0/) applies to the data made available in this article, unless otherwise stated in a credit line to the data. 
(Continued from previous page)

showed most potential (daily step count ( $p=0.098 ; 1187$ steps $[-180,2555]))$ and sedentary time $(p=0.022 ;-$ $60.8 \mathrm{~min}[-110.5,-11.0])$. However, these outcomes were poorly completed.

Conclusions: The study demonstrated that a novel social support intervention involving support from participants' close social networks, delivered via app and website, has potential to promote weight loss and is feasible and acceptable.

Trial registration: ISRCTN, ISRCTN85615983. Registered 25 September 2014

Keywords: Digital health, Obesity, Weight loss, Social support, Social network, Goal setting, Self-monitoring, Physical activity, Diet

\section{Key messages regarding feasibility}

1. What uncertainties existed regarding the feasibility?

- Was our web- and app-based healthy lifestyle intervention, that harnessed social support from friends and family, feasible to deliver and acceptable to participants?

- Would the intervention allow participants to engage social support and would this help them with weight-related lifestyle changes?

- Would our evaluation methods be feasible in a larger trial?

2. What are the key feasibility findings?

- The intervention was feasible to deliver and acceptable to participants.

- The social support aspect of the intervention was well-received and considered beneficial.

- Our evaluation methods would be feasible in a larger trial

3. What are the implications of the feasibility findings for the design of the main study?

- That this novel intervention involving social support from participants' close social networks has potential to promote weight loss and is feasible and acceptable.

\section{Background}

Poor diet, physical inactivity and high body mass index (BMI) have been highlighted in the top 10 risk factors for global burden of disease [1]. Preventive interventions which are engaging and can reach large numbers of people, including the underserved, are needed. Digital technologies have significant potential for engaging people with health behaviour change. Recent reports show that most adults in the UK, including those in socially disadvantaged groups, own a smartphone $[2,3]$.

The role of social support from family and friends is known to be important for successful weight loss and maintenance $[4,5]$. Family and friends are significant social influences on health behaviours due to factors such as intimacy, influence and proximity to day-to-day health behaviours. They are likely to be especially important at times of potential relapse. Many apps and websites have chat forums or communities of support from fellow users. There are also apps that can help connect a user with family members or friends to share stats on step count, exercise sessions, or other competitive endeavours. However, none of these apps are specifically designed to get users to engage with those closest to them to help support them in losing weight by checking on their progress and providing encouragement. This is despite existing evidence indicating the positive role of family and friends in promoting effective behaviour change rather than anonymous online contacts $[6,7]$. As far as we were aware none of these sites or apps offered the combination of elements that we used in HelpMeDoIt!, most importantly usernominated social support from key individuals within that person's social network, i.e. existing friends and family who will in many cases be the people that participants eat and exercise with.

There is also strong evidence for goal setting and selfmonitoring as successful behaviour change techniques [8-10]. These techniques derive from social cognitive theory [11] and control theory [12], the key psychological theories which inform the HelpMeDoIt! intervention, alongside social support theories. Combining social support with these techniques, using accessible, engaging technology, has the potential to impact behaviour change at a population level for low cost [13, 14]. If brief engagement with an app could mobilise existing social connections to support longer term change, then this could offer a sustainable approach to weight loss and maintenance. The main aim of the study is to explore the feasibility, acceptability and impact of a weight loss intervention, delivered via smartphone app and website, in supporting adults with obesity to achieve weight loss goals, and further to identify the value and optimal design of a future effectiveness trial.

\section{Objectives}

1. To explore the feasibility and acceptability of the intervention and its potential to reach traditionally 
underserved groups (e.g. lower socioeconomic groups)

2. To explore the barriers and facilitators to implementing the intervention

3. To investigate how participants and helpers engage with goal setting, monitoring and social support using new technologies and how these elements interact within a behaviour change intervention

4. To test the logic model and theoretical basis of the intervention

5. To investigate recruitment and retention and assess the feasibility and acceptability of outcome measures for diet and physical activity in this population

6. To use outcome data (diet, physical activity, BMI) to help decide on a primary outcome and to inform the calculation of an appropriate sample size for a full trial

7. To assess data collection tools and obtain estimates of key cost drivers to inform the design of a future cost-effectiveness analysis

8. To assess whether an effectiveness trial is warranted

\section{Methods}

\section{Design}

The study was completed in two stages: intervention development and early testing (stage 1 ) and a feasibility randomised controlled trial (stage 2).

\section{Stage 1}

Over a 12-month period, we developed and piloted the intervention. The intervention was developed iteratively with involvement of a panel of user representatives $(n=$ $10)$ and a user testing group $(n=28)$, who were recruited via advertising in local organisations, Gumtree, Twitter and Facebook. We purposively recruited a varied group in terms of age, gender, socioeconomic status and experience of using apps. All participants wanted to lose weight. Working closely with these groups, we considered how to promote engagement as well as increase the acceptability and functionality of the app and website. This early intervention development work is detailed in our full monograph [15]. We followed the UK Medical Research Council (MRC) guidance [16] and the 6SQUID [17] steps and adopted a person-centred approach [18]. At the end of the development process, we produced an updated programme theory and logic model [15] (Fig. 1 shows the final logic model from the end of the stage 2). Logic model components were directly linked to the app and website components [19], e.g. to boost motivation participants could see progress graphs, they could receive in-app medals and trophies for progress and most importantly encouragement from their helpers.

\section{Stage 2}

Stage two was a feasibility RCT, with a process evaluation and health economic component, which aimed to examine feasibility, acceptability and trial parameters for a future trial.

\section{Participants}

Participants in the Greater Glasgow and Clyde Health Board area of Scotland were eligible if they were aged 18-70, had a body mass index (BMI) of $\geq 30 \mathrm{~kg} / \mathrm{m}^{2}$, owned a smartphone and were interested in losing weight. The study had several exclusion criteria listed below:

- Terminal illness

- Previous bariatric surgery

- Dementia

- Pregnancy

- Low competence in English (inability to complete study materials)

- Contraindications to physical activity

- Participant in stage 1 intervention development

- Being a nominated helper in the trial

\section{Sample size}

This was a feasibility study and thus the main focus was to assess the acceptability of the intervention, the feasibility of the evaluation methods and to estimate parameters for a larger study. We intended to recruit 120 participants and estimated a dropout rate of $30 \%$. The final sample size of 84 for analysis was not powered to detect differences between groups for the proposed effectiveness outcomes (BMI, physical activity and diet). However, it allowed estimation of any feasibility proportion (e.g. proportions retained/found the study acceptable/provided outcome data) across the whole sample with a 95\% confidence interval of plus or minus 11 percentage points. This would also allow for the estimation of the mean of a continuous outcome (such as BMI) in the intervention arm with a $95 \%$ confidence interval of 0.262 of a standard deviation.

\section{Recruitment and randomisation}

We recruited participants in the Greater Glasgow and Clyde Health Board area of Scotland between April and October of 2016 from three sources: primary care (searched primary care databases and sent letters to potentially eligible patients), online (e.g. Gumtree, Facebook and Twitter) and community settings (e.g. advertised via local press, slimming clubs, weight management clinics, study posters in community locations) [19].

Participants were screened for eligibility and a face-toface appointment was arranged for consent and baseline data collection with a field worker. Since we were most 


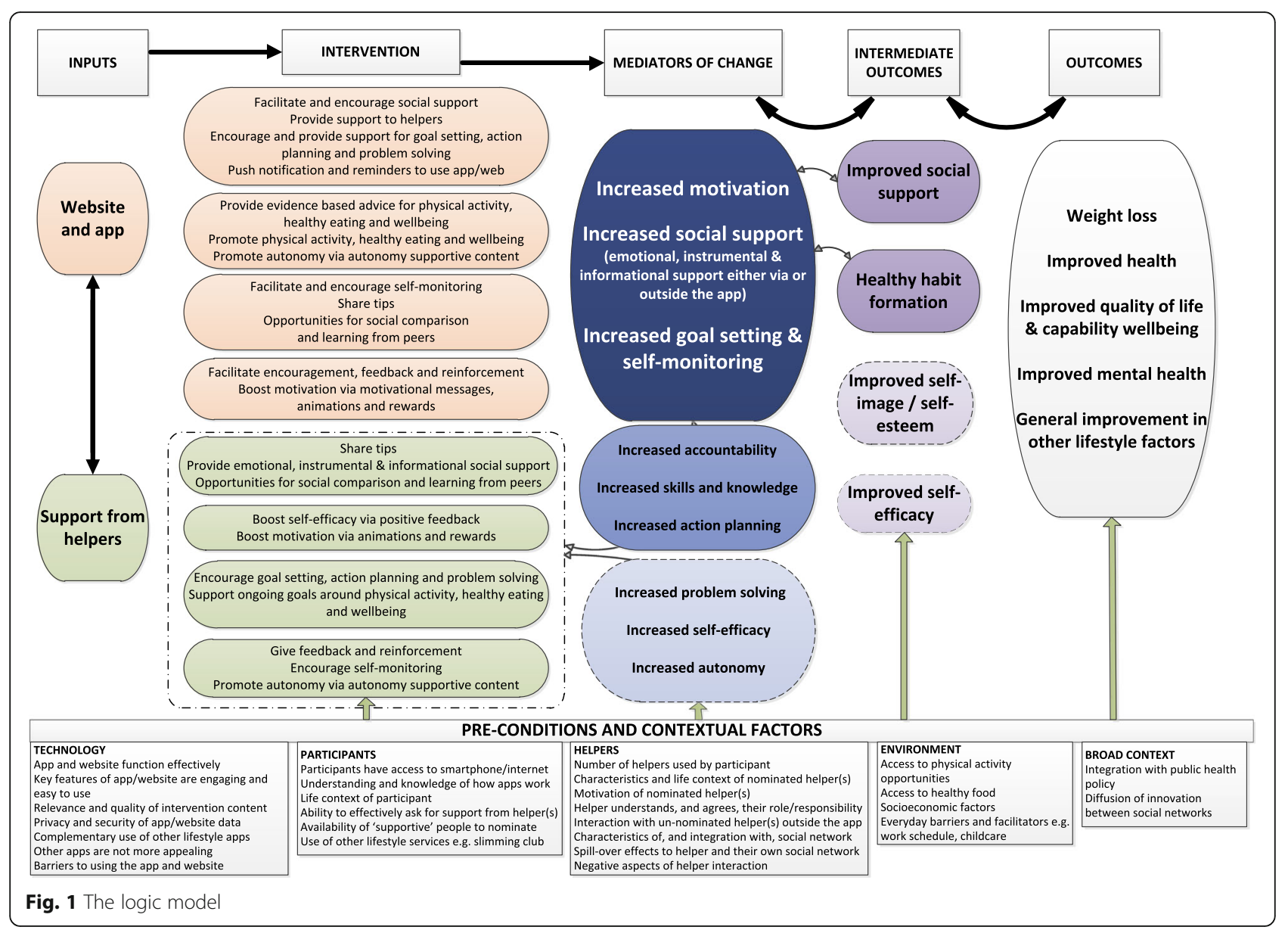

interested in the acceptability of the intervention and to improve trial efficiency, we randomised participants using a 2:1 ratio planning for 80 participants in the intervention group and 40 in the control group. Participants were allocated using a mixed randomisation/minimisation algorithm to ensure balance with respect to gender and BMI $\left(<40, \geq 40 \mathrm{~kg} / \mathrm{m}^{2}\right)$.

\section{Interventions}

The intervention group were given access to the HelpMeDoIt! app and website for 12 months. The website provided evidence-based information on weight loss, setting and monitoring goals, as well as advice on harnessing social support from family and/or friends. The app allowed participants to (i) set goals for weight loss, (ii) monitor progress and (iii) invite one or more helpers from their existing social network. This is the key novelty of this intervention whereby the app aimed to mobilise social support from people in participants' close social networks who are likely to have greater and sustained influence than, for example, those in online networks or weight loss groups. Helpers who agreed to provide support were also able to access the website and app and see participants' goals and progress. They could provide support to the participant via the app and outside of the app (e.g. face-to-face, phone call). Further details of the intervention can be found in our published protocol paper [19]. The control group received a leaflet on healthy lifestyle and were offered access to the app and website after follow-up was complete. All participants were advised that they could continue to access other sources of lifestyle change/support such as attending weight loss groups and fitness classes.

\section{Outcomes}

The key outcome of the study was whether pre-specified progression criteria were met in order to progress to a definitive trial. Feasibility and acceptability of the intervention and evaluation methods were assessed using the progression criteria outlined in Table 1 [15]. These criteria were approved by the Trial Steering Committee prior to Stage 2. Data were collected at baseline and 12 months and included (i) quantitative outcomes assessing three primary outcomes (BMI, physical activity and diet); (ii) secondary outcomes of weight, waist and hip circumference, social support, self-efficacy, motivation, mental health and health-related quality of life; (iii) qualitative interviews with participants and helpers at 6 and 12 
Table 1 Progression criteria from feasibility to full randomised controlled trial [15]

\begin{tabular}{|c|c|}
\hline Progression criterion & Method of assessment \\
\hline $\begin{array}{l}\text { 1. Are appropriate and effective routes of recruitment available to achieve a } \\
\text { powered sample size in a full trial? }\end{array}$ & $\begin{array}{l}\text { - Coming close to the sample size, as judged by the Trial Steering } \\
\text { Committee, with reasonable expectations of being able to address any } \\
\text { recruitment issues }\end{array}$ \\
\hline 2. Are participants willing to be randomised to the intervention? & $\begin{array}{l}\text { - Recruitment experiences of the study team and fieldworkers } \\
\text { - Insight from qualitative interviews with participants }\end{array}$ \\
\hline 3. Are appropriate retention rates achieved at 12-month follow-up? & $\begin{array}{l}\text { - Measured using the following scale in both the intervention and } \\
\text { control group at } 12 \text {-months: If } \geq 70 \% \text { followed-up proceed; if 50-69\% } \\
\text { followed-up discuss with Trial Steering Committee; if } \leq 49 \% \text { followed-up } \\
\text { do not proceed }\end{array}$ \\
\hline $\begin{array}{l}\text { 4. Is the intervention feasible to deliver and acceptable to participants and } \\
\text { their helpers? }\end{array}$ & $\begin{array}{l}\text { - USE questionnaire } \\
\text { - Participant/helper interviews }\end{array}$ \\
\hline $\begin{array}{l}\text { 5. Do the majority (> 50\%) of participants within the intervention group visit } \\
\text { the app at least twice OR do } 25 \% \text { of participants randomised use it three or } \\
\text { more times? }\end{array}$ & - App usage statistics \\
\hline $\begin{array}{l}\text { 6. Are identified barriers and challenges to implementation of the } \\
\text { intervention planned for and surmountable? }\end{array}$ & - Process evaluation which will present a SWOT analysis and action plan \\
\hline $\begin{array}{l}\text { 7. Do the data collection procedures effectively collect the data required for } \\
\text { a full trial? Successful completion of at least one data collection outcome } \\
\text { measure (BMI, physical activity or healthy eating) at both baseline and at } 12 \\
\text { months in those retained measured using the following scale: }\end{array}$ & $\begin{array}{l}\text { - If }>90 \% \text { of at least one data collection measure completed proceed; } \\
\text { - If } 70-89 \% \text { of at least one data collection measure completed discuss } \\
\text { strategies for improvement in future trial with Trial Steering Committee; } \\
\text { - If }<70 \% \text { of all three data collection measures completed do not } \\
\text { proceed without further modification and pilot }\end{array}$ \\
\hline 8. Are the intervention costs of a full trial covered? & - Identification of a source to pay access and treatment costs \\
\hline
\end{tabular}

months; (iv) health economic evaluation including measurement and valuation of NHS resource use, participantborne costs, intervention costs and health-related quality of life and capability wellbeing. The feasibility measures and exploratory outcome measures used are detailed in Table 2. Three primary outcomes were assessed for use in a future effectiveness trial: body mass index (BMI), physical activity and diet. We explored which of these was most feasible by assessing acceptability and data completeness. Since measuring diet [34] and physical activity [35] in community-based trials is challenging, we assessed two ways of measuring these outcomes: the DINE questionnaire [21] and 24-h dietary recall [36] for measuring diet and accelerometer [22] and 7-day physical activity recall [37] for measuring physical activity (see published protocol for details) [19].

\section{Assessment of harms}

We developed a standardized operating procedure for dealing with any reported adverse events, advised participants to discuss any health concerns with their GP and encouraged participants and fieldworkers to report negative outcomes to the study team. In addition, as part of the qualitative interviews, we explored the issue of 'harm'. The intervention was low risk to participants.

\section{Process evaluation}

The process evaluation focussed on issues related to the intervention including; context, fidelity, exposure, reach and the programme theory and logic model. Both quantitative (app and web usage statistics) and qualitative data (interviews) informed the process evaluation. At 6 months, we planned to interview up to 30 participants and 20 helpers and at 12 months up to 20 participants. Participants were purposively sampled to include variation in level of app/website use, age and gender. Semi-structured interview guides were used to explore participant insights related to acceptability of the outcome measures, acceptability and usability of the app and website, impact of the intervention on behaviour, support received from helpers and barriers to use [19]. Helper interviews focussed on intervention acceptability, the guidance provided for being a helper, types of support provided to their friend, challenges and changes in their own health behaviour as a result of being a helper. We also asked study participants about the acceptability of the outcome measures and potential contamination. Interviews were audio recorded and transcribed verbatim.

\section{Economic evaluation}

The following resource use items were collected: (i) intervention costs (fixed, variable and likely future annual estimates), (ii) primary care services provided in the NHS (e.g. GP visits, practice nurse), (iii) secondary care services provided in the NHS (e.g. A\&E attendances, hospital stay) and (iv) personal costs (e.g. household income spent on food, drinks and lifestyle activities). Medication use at baseline and follow-up was also recorded. Mean group costs were calculated by attaching the unit costs to frequency of resource use per group participant [38]. Median and ranges for resource use 
Table 2 Feasibility measures and exploratory outcomes [15]

\begin{tabular}{|c|c|c|}
\hline Measure & Method of measurement & Time-point \\
\hline \multicolumn{3}{|l|}{ Demographics } \\
\hline \multicolumn{2}{|c|}{$\begin{array}{l}\text { Case Report Form: gender, age, socioeconomic status, employment and education status, current weight } \\
\text { loss status, current health status, current computer and phone use }\end{array}$} & Baseline and 12 months \\
\hline \multicolumn{3}{|c|}{ Feasibility measures (reflecting progression criteria) } \\
\hline Recruitment & Sample size and rate of recruitment, sources of recruitment & Post baseline \\
\hline Randomisation & Interviews with participants and insight from study team & 6 months \\
\hline Retention & Retention rates for data collection at 12 months follow-up & 12 months \\
\hline $\begin{array}{l}\text { Feasibility of app/website } \\
\text { (intervention) }\end{array}$ & $\begin{array}{l}\text { Interviews with participants, interviews with helpers, app and website } \\
\text { usage statistics, USE [20] questionnaire }\end{array}$ & $\begin{array}{l}6 \text { and } 12 \text { months, } 12 \text { months, } 12 \\
\text { months and } 12 \text { months }\end{array}$ \\
\hline Data collection & Rates of completion for different measures & Baseline and 12 months \\
\hline \multicolumn{3}{|l|}{ Exploratory primary outcomes } \\
\hline $\mathrm{BMI}\left(\mathrm{kg} / \mathrm{m}^{2}\right)$ & Physical measurement of height $(\mathrm{m})$ and weight $(\mathrm{kg})$ & Baseline and 12 months \\
\hline Diet & DINE questionnaire [21] (via telephone), 4 days of 24-h dietary recall & Baseline and 12 months \\
\hline Physical activity & 7 day accelerometry [22], 7 day Physical Activity Recall Questionnaire [23] & Baseline and 12 months \\
\hline \multicolumn{3}{|l|}{ Secondary outcomes } \\
\hline Anthropometric changes & Waist and hip circumference (cm) & Baseline and 12 months \\
\hline Health-related quality of life & EQ5D 3 -L questionnaire [24], ICECAP A scale [25] & Baseline and 12 months \\
\hline Mental health & General Health Questionnaire (GHQ12) [26] & Baseline and 12 months \\
\hline $\begin{array}{l}\text { NHS resource use and } \\
\text { participant-borne costs }\end{array}$ & Specially designed resource use questionnaire & Baseline and 12 months \\
\hline Social support & Exercise and Eating Habits Social Support Scales [27] & Baseline and 12 months \\
\hline Self-efficacy & Weight [28] and Exercise Efficacy Lifestyle Scales $[29,30]$ & Baseline and 12 months \\
\hline Motivation & Treatment Self-Regulation Questionnaire [31] & Baseline and 12 months \\
\hline Smoking use & Heaviness of Smoking Index (HIS) [32] & 12 months \\
\hline Alcohol use & Alcohol Use Disorders Identification Test (AUDIT-C) [33] & 12 months \\
\hline
\end{tabular}

quantities and costs are reported. The feasibility of using the EuroQol EQ5D-3L [24] instrument and the ICECAP-A [25] instrument as a means of capturing any short term effects on health-related quality of life or capability wellbeing was assessed. This involved exploring data completeness and response rates. Participant responses for both instruments were converted to a utility score using the value set elicited from UK general population.

\section{Main analyses}

Baseline characteristics were summarised. Feasibility measures were the primary focus of the analysis. Rates of recruitment, randomisation and retention at 12 months follow-up, were calculated with $95 \%$ confidence intervals. Usage data for the app and website was explored, and a range of summary measures presented.

Exploratory outcomes were analysed using linear regression models, with randomised group, the baseline measurement of the outcome, age, gender and BMI as predictor variables. Model residuals were assessed for normality, and where necessary, outcome measures (at follow-up and at baseline) were transformed to improve model fit. All analyses were conducted under intention to treat principles. Complete case analysis was used, unless more than $20 \%$ of cases were lost due to missing data, in which case multiple imputation was also performed. These analyses were exploratory and underpowered, so no formal hypothesis testing was performed. $p$ values are presented for descriptive purposes, as a guide to the interpretation of the magnitude of reported associations. Effect sizes were calculated by dividing intervention effect estimates by the pooled standard deviation of the change from baseline in the outcome measure, and reported in line with CONSORT guidelines for reporting feasibility and pilot studies [39].

\section{Health economic analyses}

The health economics cost data were analysed as follows: Resource use data were summarised and described using mean values and variation around these estimates. Key fixed and variable costs of developing the intervention were described and summarised. EQ5D-3 L [24] and ICECAP-A [25] outcome data were reported by 
within-attribute response rates, mean values and associated variance. Within-trial economic analyses were performed using STATA 12.0 (StataCorp, TX, USA).

\section{Qualitative analyses}

Qualitative data were analysed by two researchers who independently coded using thematic analysis [40, 41]. Twenty percent of the interviews were double coded by two researchers who resolved disagreements by discussion. The coding framework was discussed between the researchers and also within the wider study team to finalise the themes and sub-themes. The results of the qualitative analyses were combined with intervention usage data to explore and refine the HelpMeDoIt! programme theory, in order to better understand the mechanisms and key contextual factors to consider when refining the intervention and evaluation design.

\section{Results}

109 participants were recruited to the HelpMeDoIt! trial (Fig. 2) and randomised 2:1 to the intervention $(n=73)$ and control group $(n=36)$. Baseline characteristics

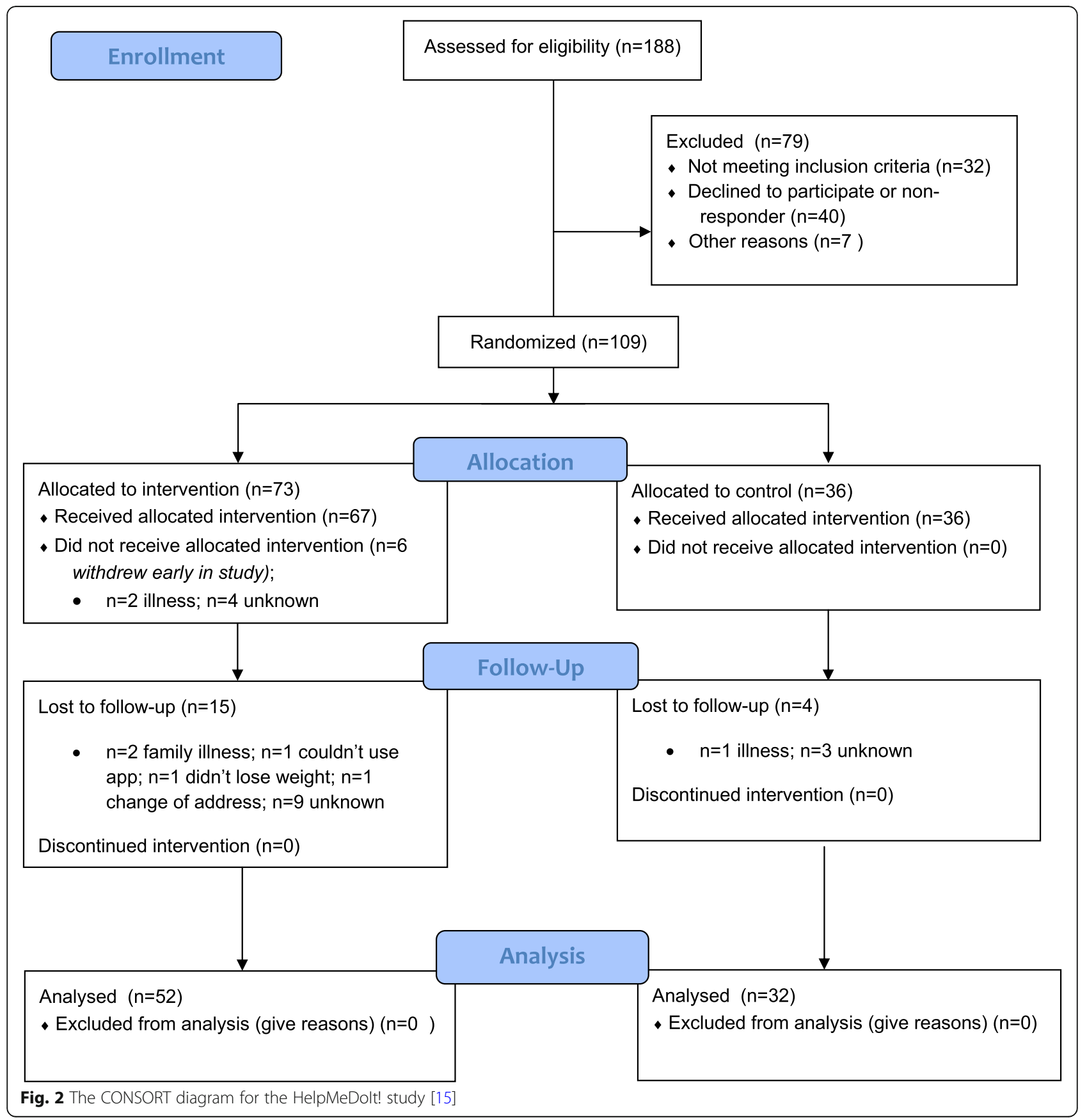


included 69.7\% ( $n=73)$ women, mean age of 47 (range 25-68), mean BMI $37.6 \mathrm{~kg} / \mathrm{m}^{2}$ and $36 \%$ were from the highest quintile (most deprived) of socioeconomic deprivation (Table 3) [15]. Key findings are presented below, with additional detailed findings published in our monograph [15].

\section{Progression criteria}

The pre-specified progression criteria (Table 1) were achieved [15]. It was feasible to recruit and retain participants in the trial (progression criteria 1-3). Our target sample size was 120 participants. Our recruitment was hampered by a 2-month delay in gaining governance approvals. We recruited 109 participants over a 6-month period (91\% of our target). In order to keep within the study timeline we had to stop recruitment at 6 months.
This was just short of our target which would likely have been achieved if part of our recruitment had avoided the summer holiday months. The slightly smaller sample size was not an issue in terms of the study aims, in order to assess the acceptability and feasibility of the intervention and evaluation methods the sample size we recruited was sufficient. At 12 months we achieved a follow-up rate of $77.1 \%$ (84 of 109 participants). Followup rates were different between intervention and control groups ( $71 \%$ and $89 \%$, respectively). We developed an intervention that was feasible to deliver and acceptable to helpers and participants (see the 'Process evaluation findings' section data) (progression criteria 4). Two thirds of intervention participants (including those who withdrew from the study) visited the app twice or more, and $52 \%$ visited three times or more (progression criteria

Table 3 Baseline characteristics of randomised participants [15]

\begin{tabular}{|c|c|c|c|c|}
\hline & & All & Intervention & Control \\
\hline N & & 109 & 73 & 36 \\
\hline Participant age (years) & Mean (SD) & $47.3(10.7)$ & $46.2(10.6)$ & $49.4(10.7)$ \\
\hline \multirow[t]{2}{*}{ Participant sex } & N (\%) Female & $76(69.7)$ & $49(67.1)$ & $27(75.0)$ \\
\hline & $N(\%)$ Male & $33(30.3)$ & $24(32.9)$ & $9(25.0)$ \\
\hline \multirow[t]{5}{*}{ SIMD quintile ${ }^{a}$} & N (\%) Q1—most deprived & $36(36.4)$ & $25(37.9)$ & $11(33.3)$ \\
\hline & $N(\%) \mathrm{Q} 2$ & $21(21.2)$ & $15(22.7)$ & $6(18.2)$ \\
\hline & N (\%) Q3 & $13(13.1)$ & $7(10.6)$ & $6(18.2)$ \\
\hline & $N(\%) \mathrm{Q} 4$ & $16(16.2)$ & $10(15.2)$ & $6(18.2)$ \\
\hline & N (\%) Q5-least deprived & $13(13.1)$ & $9(13.6)$ & $4(12.1)$ \\
\hline \multirow[t]{2}{*}{ Marital status $^{b}$} & $N(\%)$ Living with partner & $61(59.2)$ & $41(60.3)$ & $20(57.1)$ \\
\hline & $N(\%)$ Single & $42(40.8)$ & $27(39.7)$ & $15(42.9)$ \\
\hline \multirow[t]{6}{*}{ Ethnicity } & N (\%) White British//rish & $91(84.3)$ & $57(79.2)$ & $34(94.4)$ \\
\hline & $N(\%)$ White Other & $6(5.6)$ & $5(6.9)$ & $1(2.8)$ \\
\hline & $N(\%)$ Indian & $2(1.9)$ & $1(1.4)$ & $1(2.8)$ \\
\hline & N (\%) Pakistani & $2(1.9)$ & $2(2.8)$ & $0(0.0)$ \\
\hline & N (\%) Chinese & $1(0.9)$ & $1(1.4)$ & $0(0.0)$ \\
\hline & $N(\%)$ Other & $6(5.6)$ & $6(8.3)$ & $0(0.0)$ \\
\hline \multirow[t]{2}{*}{ Education ${ }^{c}$} & $N(\%)$ Higher education & $64(61.5)$ & $47(67.1)$ & $17(50.0)$ \\
\hline & $N(\%)$ Other & $40(38.5)$ & $23(32.9)$ & $17(50.0)$ \\
\hline \multirow[t]{3}{*}{ Employment } & N (\%) Employee & $86(78.9)$ & $57(78.1)$ & 29 (80.6) \\
\hline & $N(\%)$ Self-employed & $16(14.7)$ & $12(16.4)$ & $4(11.1)$ \\
\hline & N (\%) Not employed & $7(6.4)$ & $4(5.5)$ & $3(8.3)$ \\
\hline Access to computer at home & $N(\%)$ & $103(95.4)$ & $68(94.4)$ & $35(97.2)$ \\
\hline Use internet every day & $N(\%)$ & $105(97.2)$ & $69(95.8)$ & $36(100.0)$ \\
\hline Weight (kg) & Mean (SD) & $104.6(20.7)$ & $105.7(21.4)$ & $102.2(19.4)$ \\
\hline $\mathrm{BMI}\left(\mathrm{kg} / \mathrm{m}^{2}\right)$ & Mean (SD) & $37.6(5.9)$ & $37.8(6.0)$ & $37.1(5.7)$ \\
\hline Waist circum. (cm) & Mean (SD) & $114.1(14.7)$ & $113.9(15.4)$ & $114.6(13.2)$ \\
\hline Hip circum. (cm) & Mean (SD) & $124.3(13.4)$ & $125.0(14.2)$ & $122.7(11.7)$ \\
\hline
\end{tabular}

${ }^{\mathrm{a}} \mathrm{SIMD}=$ Scottish Index of Multiple Deprivation

${ }^{\mathrm{b}}$ Living with partner $=$ married/civil partnership/cohabiting; Single $=$ single/widowed/divorced

${ }^{c}$ Higher education $=$ higher degree/first degree/certificate/diploma; Other $=\mathrm{A}$ or AS/O levels/other 
5). See the 'Process evaluation findings' for further usage data. Data collection methods were feasible to use except for the 24-h multiple pass recall dietary measure, which was poorly completed at baseline and therefore not used at follow-up, and issues with obtaining valid accelerometry data (progression criteria 7). Barriers and challenges to implementation have been planned for and are surmountable (progression criteria 6). This is discussed further below.

\section{Process evaluation findings}

A summary of the key findings from the process evaluation is given below with further details published elsewhere [15]. Interviews were conducted with 35 individuals (22 participants and 9 helpers at 6 months and 4 participants at 12 months). Most study participants interviewed were positive and engaged with the HelpMeDoIt! intervention. All study participants used a helper to support them with weight loss. Many of these helpers were nominated through the app; however, for some participants, this was done outside of the app. Social support was a key element, with helpers providing emotional, informational and instrumental support to participants. The emotional support and encouragement from helpers were seen as key. Many participants set goals via the app (mean 13.1 range 0.143 ) for healthy eating, physical activity and other behaviours. Participants reported monitoring their progress toward goals and also using other apps for self-monitoring. Sixty-one percent of the 954 goals created by participants were completed. Participants who were most successful at losing weight had varied and good social support and were positive about the goal setting and self-monitoring.

Helpers described how they enjoyed supporting their friend with their weight loss goals. They reported receiving mutual support with their own lifestyle goals and that they were more motivated to eat well and be active. Few helpers used the app, they reported technical difficulties, lacking confidence with smartphones, or preferring to support their friend outside of the app, e.g. face-to-face interactions. They believed their support contributed to their friend's motivation to make healthy changes.

Interview findings helped inform refinement of the programme theory and logic model. Motivation was identified as a key mediator influencing behaviour and encouragement from the helpers was important to boost motivation. Participants reported positive lifestyle changes in both their helpers and their broader social network. Contextual factors like mood or significant life changes were reported as influencing participants' engagement with the intervention. Factors highlighted for consideration in future work included difficulty asking friends/relatives for support, lack of available support, social and group norms related to food and exercise and personal barriers to lifestyle, such as motivation.

Despite a 3-month testing phase, there were initial technical issues with the app. The majority of dissatisfaction and barriers to use were related to these issues. The app underwent a 'rebuild' which resolved the software problems. Participants who used the app most frequently, once the technical issues were resolved, provided the most positive feedback via both qualitative and quantitative measures. Of the 54 (74\%) participants who downloaded the app, $48(89 \%)$ used it twice or more. In total, 45 helpers were invited, ranging from 1 to 8 helpers per participant. Of the 45 invited helpers, $25(56 \%)$ accepted the invitation and downloaded the app. Most helpers did not engage with the app on a frequent basis. However, interview data indicated that helpers were sometimes unclear how to use the app to help their family or friend, with many providing support through face-to-face interactions instead. While participants and helpers did not access the website regularly, it is likely that one or two visits would be enough to get the information needed. The app also delivered the key information from the website via push notifications and daily messages.

Although not part of the process evaluation, we asked participants about the trial methods. They were positive about the evaluation methods, such as the data collection measures and retention strategies and there was no evidence of contamination in the data.

\section{Exploratory outcomes}

The feasibility trial was not powered to detect statistically significant changes, but to explore the feasibility and sensitivity of measures for use in a definitive trial. Three outcomes were assessed: BMI, physical activity and diet. BMI was successfully measured in $98 \%$ of the sample ( $82 \%$ objectively and $16 \%$ via self-report) and diet (DINE questionnaire) in 96\% (81 of 84). Physical activity data were successfully collected via the self-report 7-day physical activity recall from $96 \%$ of participants. However, objective accelerometry was only available from $46 \%$ of participants. The secondary outcomes were feasible and acceptable to use.

Objective physical activity data showed moderate to large effect size estimates across several measures, particularly the daily step count and sedentary time (Table 4). There was no evidence to suggest that self-report physical activity was different between those who did and did not provide valid accelerometry data, thereby increasing confidence in these results. However, these outcomes were poorly completed with only 24 in the intervention arm and 15 in the control providing valid data. Both methods suggested a decrease in physical 
Table 4 Measures of BMI, physical activity and dietary outcome (DINE) at baseline and at 12-month follow-up for the subset of participants providing data at both time points [15]

\begin{tabular}{|c|c|c|c|c|c|c|c|}
\hline & \multirow[t]{2}{*}{$N$} & \multirow[t]{2}{*}{ Baseline } & \multirow[t]{2}{*}{12 months } & \multirow[t]{2}{*}{ Change } & \multicolumn{3}{|c|}{ Between-group difference (Int ${ }^{n}-$ Control) } \\
\hline & & & & & & Estimate $(95 \% \mathrm{Cl})$ & ES $(95 \% \mathrm{Cl})$ \\
\hline \multicolumn{8}{|c|}{ BMI (kg/m2) } \\
\hline Con & 32 & $36.9(5.7)$ & $36.0(6.3)$ & $-0.9(3.3)$ & Unadj. & $-0.3(-1.5$ to 0.9$)$ & $\begin{array}{l}-0.11(-0.56 \text { to } \\
0.33)\end{array}$ \\
\hline Int & 50 & $36.9(5.3)$ & $35.7(5.4)$ & $-1.2(2.4)$ & Adj. & $-0.2(-1.4$ to 1.0$)$ & $\begin{array}{l}-0.08(-0.52 \text { to } \\
0.37)\end{array}$ \\
\hline \multicolumn{8}{|c|}{ MVPA time as $\%$ of wear time (from activity monitor) ${ }^{*}$} \\
\hline Con & 15 & $4.1(2.3)$ & $3.5(2.3)$ & $-0.6(1.0)$ & Unadj. & $-0.3(-2.2,1.7)$ & $-0.08(-0.73,0.56)$ \\
\hline Int & 24 & $6.5(4.0)$ & $5.6(2.5)$ & $-0.9(3.8)$ & Adj. & $1.3(-0.1,2.7)$ & $0.44(-0.02,0.90)$ \\
\hline \multicolumn{8}{|c|}{ Average daily MVPA time (min, from activity monitor) } \\
\hline Con & 15 & $35.5(19.4)$ & $31.3(20.3)$ & $-4.3(10.5)$ & Unadj. & $-4.2(-21.1,12.7)$ & $-0.16(-0.81,0.48)$ \\
\hline Int & 24 & $54.8(34.3)$ & $46.3(20.2)$ & $-8.5(32.2)$ & Adj. & $9.3(-2.3,20.9)$ & $0.35(-0.09,0.80)$ \\
\hline \multicolumn{8}{|c|}{ Average daily sedentary time (min, from activity monitor) } \\
\hline Con & 15 & $\begin{array}{l}661.7 \\
(138.2)\end{array}$ & $\begin{array}{l}703.4 \\
(166.6)\end{array}$ & $41.7(83.5)$ & Unadj. & $-52.9(-104.4,-1.4)$ & $-0.66(-1.31,-0.02)$ \\
\hline Int & 24 & $642.7(94.0)$ & $631.5(82.8)$ & $-11.2(77.6)$ & Adj. & $\begin{array}{l}-60.8(-110.5,- \\
11.0)\end{array}$ & $-0.76(-1.38,-0.14)$ \\
\hline \multicolumn{8}{|c|}{ Average daily step count (from activity monitor) } \\
\hline Con & 15 & $5650(1526)$ & $5335(1844)$ & $\begin{array}{l}-315 \\
(1130)\end{array}$ & Unadj. & $43(-1876,1963)$ & $0.01(-0.63,0.66)$ \\
\hline Int & 24 & $7232(3712)$ & $6960(2568)$ & $\begin{array}{l}-272 \\
(3669)\end{array}$ & Adj. & $1187(-180,2555)$ & $0.40(-0.06,0.86)$ \\
\hline \multicolumn{8}{|c|}{ Average daily energy expenditure (kcal/day, self-report) } \\
\hline Con & 32 & $3879(1121)$ & $3606(750)$ & $-273(828)$ & Unadj. & $113(-179,404)$ & $0.17(-0.27,0.62)$ \\
\hline Int & 49 & $3717(715)$ & $3557(779)$ & $-160(502)$ & Adj. & $62(-180,304)$ & $0.09(-0.28,0.47)$ \\
\hline \multicolumn{8}{|c|}{ Average daily energy expenditure per kg body weight (kcal/kg/day, self-report) } \\
\hline Con & 32 & $38.2(7.1)$ & $36.7(4.1)$ & $-1.6(6.5)$ & Unadj. & $1.0(-1.6,3.6)$ & $0.17(-0.27,0.62)$ \\
\hline Int & 49 & $37.2(4.9)$ & $36.7(3.7)$ & $-0.6(5.3)$ & Adj. & $0.0(-1.7,1.6)$ & $0.00(-0.29,0.28)$ \\
\hline \multicolumn{8}{|c|}{ Fibre score (score < 30 = low fibre) } \\
\hline Con & 32 & $18.6(10.6)$ & $19.2(11.6)$ & $0.6(12.6)$ & Unadj. & $-4.1(-9.2,0.9)$ & $-0.36(-0.81,0.08)$ \\
\hline Int & 49 & $19.8(9.7)$ & $16.3(11.8)$ & $-3.6(10.6)$ & Adj. & $-3.3(-8.1,1.5)$ & $-0.29(-0.71,0.13)$ \\
\hline \multicolumn{8}{|c|}{ Fat score (score < 30 = low fat) } \\
\hline Con & 32 & $27.1(12.6)$ & $22.9(9.6)$ & $-4.1(10.8)$ & Unadj. & $-1.4(-6.4,3.6)$ & $-0.13(-0.57,0.32)$ \\
\hline Int & 49 & $28.4(11.6)$ & $22.9(12.7)$ & $-5.5(11.4)$ & Adj. & $-0.4(-4.8,4.0)$ & $-0.04(-0.43,0.36)$ \\
\hline \multicolumn{8}{|c|}{ Healthy eating score (score = fibre - fat; negative score indicates unhealthy diet) } \\
\hline Con & 32 & $-8.5(16.1)$ & $-3.8(13.2)$ & $4.7(16.6)$ & Unadj. & $-2.7(-8.9,3.4)$ & $-0.20(-0.64,0.25)$ \\
\hline Int & 49 & $-8.6(13.3)$ & $-6.6(12.9)$ & $2.0(11.7)$ & Adj. & $-2.9(-8.0,2.2)$ & $-0.21(-0.58,0.16)$ \\
\hline \multicolumn{8}{|c|}{ Unsaturated fat score (score 6-9 = moderate unsaturated fat intake) } \\
\hline Con & 32 & $9.3(1.8)$ & $8.2(3.7)$ & $-1.1(3.5)$ & Unadj. & $-2.3(-4.3,-0.3)$ & $-0.45(-0.85,-0.06)$ \\
\hline Int & 49 & $9.2(2.2)$ & $5.8(5.0)$ & $-3.3(5.6)$ & Adj. & $-2.2(-4.0,-0.4)$ & $-0.44(-0.80,-0.07)$ \\
\hline \multicolumn{8}{|c|}{ Fruit and vegetable score (score = portions per day) } \\
\hline Con & 32 & $5.0(3.4)$ & $4.9(2.9)$ & $-0.1(3.4)$ & Unadj. & $0.7(-0.6,1.9)$ & $0.24(-0.21,0.69)$ \\
\hline Int & 49 & $4.7(2.6)$ & $5.2(2.5)$ & $0.5(2.2)$ & Adj. & $0.4(-0.6,1.5)$ & $0.16(-0.23,0.55)$ \\
\hline \multicolumn{8}{|c|}{ Fizzy juice score (score = cans of juice per day) } \\
\hline Con & 32 & $0.2(0.6)$ & $0.3(0.9)$ & $0.1(0.4)$ & \multirow{2}{*}{\multicolumn{2}{|c|}{ Non-parametric test ${ }^{\mathrm{a}}$}} & \\
\hline Int & 49 & $0.0(0.0)$ & $0.2(0.6)$ & $0.2(0.6)$ & & & \\
\hline \multicolumn{8}{|c|}{ Sugar score (score = tsps of sugar per day) } \\
\hline Con & 32 & $0.9(5.3)$ & $0.8(3.5)$ & $-0.2(1.9)$ & \multirow{2}{*}{\multicolumn{2}{|c|}{ Non-parametric test ${ }^{\mathrm{a}}$}} & \\
\hline Int & 49 & $0.5(1.3)$ & $0.3(0.8)$ & $-0.2(0.9)$ & & & \\
\hline
\end{tabular}

Models adjusted for baseline value, age, gender and high BMI (>40) 
activity from baseline, with accelerometry showing a decrease in both groups for MVPA, and self-report showing a decrease in both groups for energy expenditure. With regard to the diet scores, there was low power and no consistent patterns in terms of betweengroup differences (Table 4).

Both groups showed similar reductions in BMI during the study of $-1.2 \mathrm{~kg} / \mathrm{m}^{2}$ (SD 2.4) in the intervention group, and $-0.9 \mathrm{~kg} / \mathrm{m}^{2}$ (SD 3.3) in the control group. Mean kg weight loss for intervention participants was $3.3 \mathrm{~kg}$ (SD 6.5) compared with $-2.5 \mathrm{~kg}$ (SD 9.3) for control participants. Mean \% weight loss for intervention participants was $-3.2 \%$ (SD 6.2\%), and $-2.3 \%$ (SD 8.7\%) for control participants (Table 4).

Sixty-six percent of 50 intervention group participants for whom weight was measured at both baseline and follow-up had lost weight, compared to 53\% of 32 participants in the control group $(p=0.26)$. These analyses were exploratory and underpowered to detect effects. However, for the key weight-related outcomes of interest, the confidence intervals were generally wide and therefore consistent with clinically relevant benefits.

\section{Economic evaluation}

Total study intervention costs (fixed and variable) included app development costs of $£ 60,000$ and incentive/ retention payments of $£ 4360$ (vouchers, newsletters). Depending upon throughout and lifespan, 'per participant' cost will vary by study. The resource use patterns were similar across groups; the main items of resource use were GP, practice nurse, physiotherapist, A\&E and hospitalisations. The cost of lifestyle activities in the 3 months prior to follow-up was in the range $£ 50-55$. Mean weekly cost of food and drink at follow-up was in the range of $£ 75-100$ across both groups with most food expenditure on groceries followed by meals out, take away and alcohol spend. This pattern was the same across both arms, at baseline and follow-up. Both QOL and capability wellbeing questionnaires were completed by participants at baseline and 12 months follow-up. At 12 months, 78 participants completed the EQ5D-3L [24], ICECAP-A [25] and expenditure on food purchases. Only 69 provided data on health, social and personal resource use. This was because a few participants at risk of dropping out of the study were offered a 'minimum data set' that omitted the additional questions linked to resource use. The EQ5D and associated visual analogue scale results revealed the typical 'healthy population' values of around $0.7-0.8$ and revealed expected variation in values between the measures. There were no implausible data for the EQ5D_3L. Full economic findings, including medication use and mean group costs, can be found in our published monograph [15].
Overall, the results of the economic evaluation feasibility study showed that the questionnaires designed for measuring resource use, lifestyle, grocery and alcohol spend, health-related quality of life and capability would be suitable for inclusion in a full study with some minor re-design of the resource use questions.

There were no serious adverse events reported during the study.

\section{Discussion}

Family and friends are likely to be a key influence on weight-related health behaviours $[4,5]$, since these are the people whom we are closest to, spend most time with and with whom we are likely to eat and exercise with. For these reasons, they are likely important not only for initiation of behaviour change, but also for longer term maintenance. Although other apps/websites develop user communities that offer social support, they are unlikely to have such a large and sustained influence as family and friends. This study assessed the feasibility and acceptability and impact of a novel, theory-informed weight loss intervention that combined evidence-based behaviour change techniques with mobilising social support from a participant's close social network.

Overall, the study findings were positive indicating the intervention and trial methods were feasible and acceptable and both the qualitative and quantitative results indicated the intervention had potential. Most effect size estimates had confidence intervals that included 0.5 in favour of the intervention, which would generally be considered a moderate effect size for a low-cost intervention of this nature. Although MVPA and energy expenditure decreased in both groups, participants were relatively active at baseline, with the intervention arm achieving an average of $50 \mathrm{~min}$ and controls an average of $36 \mathrm{~min}$ of moderate to vigorous physical activity per day. It should also be noted that studies have found that people with obesity are likely to over-estimate their physical activity using self-report measures [42]. In addition, all key progression criteria were achieved, recruitment rates were adequate ( $91 \%$ of target), retention was good ( $77.1 \%$ at 12 -months) and engagement with the intervention was acceptable.

Qualitative data supported the programme theory of the intervention (Fig. 1) [15], since the app facilitated engaging support from people that participants already knew to help their weight loss attempts. The findings supported the key role of social support from existing social networks. Motivation, goal setting and selfmonitoring were supported as core elements of the programme theory. The app was a catalyst to engaging social support either via the app or outside of the app. It is likely that a number of participants in the study who enrolled or downloaded the app did not engage or 
benefit from the intervention. However, this does not undermine the potential reach and cost effectiveness, as there is some evidence that the app may be useful for some individuals and this is a low-cost way to facilitate social support from close social networks, which is known to be effective in assisting and maintaining behaviour change.

The low engagement with the website by both participants and helpers suggests a need for better signposting, linking to website information within the app, or perhaps that the website is not needed. There was also low engagement of helpers via the app. Technical issues with the app in the early stages of the trial may have led to participants disengaging with the app and the trial. Adherence to the intervention was superior to that seen in general app usage [43] and similar to that seen in other studies testing behaviour change apps [44, 45]. An expert international workshop concluded that engagement with apps is complex and is more than how often a person simply uses an app [46]. Continued engagement with a digital intervention (app/website) is not always needed for behaviour change, as initial exposure to a digital intervention could be enough to kick start a process of establishing new skills and habits [46]. This is particularly relevant to HelpMeDoIt!, where a brief interaction with the website or app could lead to the engagement of significant and sustained social support from existing, stable and durable social resources. The effects of this support on resultant behaviour are not dependent on further app use.

The study had a number of key strengths. The intervention was developed with substantial input from potential users and was developed using recommended frameworks for developing complex interventions and digital interventions. The intervention was theory-based and informed by current evidence regarding successful behaviour change techniques for weight loss. We used a multiple methods approach and were able to triangulate these data to strengthen the internal and external validity of the findings.

The feasibility trial used rigorous methods for data collection and analyses. Objective measures were used to assess weight, BMI and physical activity, and self-report measures were chosen based on previous evidence of validity and reliability. The study also included measures of quality of life and capability wellbeing and collected cost data to inform a future cost-effectiveness analysis. We recruited a clinically important sample with a mean BMI of $37.6 \mathrm{~kg} / \mathrm{m}^{2}$ (SD 5.9). We were particularly successful in recruiting participants from lower socioeconomic groups with over a third from the highest quintile of deprivation in Scotland and a further 21\% from the next most deprived quintile. This is important as research has shown that it is often difficult to recruit and engage participants from lower socioeconomic groups in research [47] and many current interventions widen inequalities [48]. Finally, the qualitative methods were robust, and the large number of interviews gave extensive, in-depth accounts of the experiences of both the study participants and their helpers. The findings from the process evaluation informed refinements of the programme theory, logic model and trial methods in anticipation of a future effectiveness trial.

Limitations of the study included ethical constraints during the stage 2 follow-up, which meant that we were unable to invite helpers directly to be interviewed. Because of this, the number of helper interviews was smaller than anticipated. Only about half the participants had complete accelerometry data. A wrist worn accelerometer may have had better compliance. In other similar studies, strategies like vouchers for return of the accelerometers have led to improved adherence and return rates, so vouchers combined with other methods could significantly improve return rates in a future trial $[49,50]$.

While the aim of this research was to develop an intervention that maximised effect size and participation, an intervention like this could have high reach and therefore tolerate small effects and low usage overall while remaining cost-effective. For some people, meaningful engagement will be catalysed by the app for whom the effects should be worthwhile. It will also be important to consider the contribution of such mHealth approaches within the wider ecological public health context for weight management and sustained weight reduction. If the intervention was found to be effective in a larger trial, then HelpMeDoIt! may have the potential to deliver a low-cost, high-reach intervention for adults with obesity, including those in socioeconomically disadvantaged groups. It could be used as a complementary intervention used alongside other health care or lifestyle services. HelpMeDoIt! may have potential to positively influence the lifestyle of individuals in a participants' broader social network. This approach to mobilising social support for health behaviour change could be used in other lifestyle behaviours or used as part of other app-based interventions. Key areas of future work include further exploration of the key mechanisms of change as well as the motivation and engagement of helpers in relation to providing social support to participants and then after some refinement, assessment of the effectiveness and cost-effectiveness of the HelpMeDoIt! intervention.

\section{Acknowledgements \\ We would like to thank the study participants and the Population Health Research Facility staff for their help in supporting the study.}

\section{Authors' contributions}

SS conceived the study and wrote the first draft of this manuscript. LM and

JP contributed to writing the manuscript and analysed the process 
evaluation data. EM conducted the economic evaluation and drafted the relevant sections. AM and MK contributed to the study design, completed the statistical analyses and drafted those sections. SMT assisted with qualitative methods and study design. OUM was a major contributor in delivering stage 1 of the research. LARM contributed to the study design and edited the manuscript. $\mathrm{KH}$, EC and SM contributed to the study design. All authors read, commented on and approved the final manuscript.

\section{Funding}

National Institute of Health Research: Public Health Research Programme 12/ 180/20. Additional time on the study was supported by UK Medical Research Council and Chief Scientist Office core funding as part of the MRC/CSO Social and Public Health Sciences Unit 'Complexity in Health Improvement' programme (MC_UU_12017/14 and SPHSU14) and 'Social Relationships and Health Improvement' programme (MC_UU_12017/11 and SPHSU11). SS was further supported by MRC Strategic Award MC_PC_13027.

\section{Availability of data and materials}

The datasets used and/or analysed during the current study are available from the corresponding author on reasonable request.

\section{Ethics approval and consent to participate}

Stage 1 ethical approval was granted by the University of Glasgow Medical, Veterinary and Life Sciences College Ethics Committee (reference 200140108). Stage 2 ethical approval was granted by the NHS West of Scotland Research Ethics Committee (reference 15/WS/0288). Research governance approval was granted by NHS Greater Glasgow and Clyde Health Board.

\section{Consent for publication}

Not applicable

\section{Competing interests}

EM declares she is a member of the NIHR PHR funding board. SS declares that she is a member of the NIHR Clinical Evaluation and Trials committee, the NIHR Policy Research Programme committee, the Chief Scientist Office Health Improvement, Protection and Services Research Committee. The remaining authors declare that they have no competing interests.

\section{Author details}

${ }^{1}$ MRC/CSO Social and Public Health Sciences Unit, Institute of Health and Wellbeing, University of Glasgow, Berkeley Square, 99 Berkeley Street, Glasgow G3 7HR, UK. ${ }^{2}$ Robertson Centre for Biostatistics, Institute of Health and Wellbeing, University of Glasgow, Robertson Centre, Boyd Orr Building, Glasgow G12 8QQ, UK. ${ }^{3}$ Health Economics and Health Technology Assessment Unit (HEHTA), Institute of Health and Wellbeing, University of Glasgow, 1 Lilybank Gardens, Glasgow G12 8RZ, UK. ${ }^{4}$ Centre for Trials Research, Cardiff University, Neuadd Meirionnydd, Heath Park Way, Cardiff CF14 4YS, UK. ${ }^{5}$ Division of Population Medicine, School of Medicine, Cardiff University, Neuadd Meirionnydd, Heath Park Way, Cardiff CF14 4YS, UK. ${ }^{6}$ College of Engineering, Mathematics and Physical Sciences, School of Mathematics/The Alan Turing Institute, University of Exeter, Harrison Building, Streatham Campus, North Park Road, Exeter EX4 4QF, UK. ' Institute of Health Research, College of Medicine and Health, University of Exeter, College House, St Luke's Campus, Heavitree Road, Exeter EX1 2LU, UK. ${ }^{8}$ Centre for the Development and Evaluation of Complex Interventions for Public Health Improvement (DECIPHer), Cardiff School of Social Sciences, Cardiff University, Cardiff CF10 3AT, UK.

\section{Received: 19 February 2020 Accepted: 30 July 2020}

Published online: 19 September 2020

\section{References}

1. World Health Organization. Global Health Risks: mortality and burden of disease attributable to selected major risks. Geneva: World Health Organization; 2009.

2. Ofcom. The Communications Market 2018: Summary 2018 [cited 201912 November]. Available from: https://www.ofcom.org.uk/research-and-data/ multi-sector-research/cmr/cmr-2018/summary.

3. Scottish Government. Scotland's People Annual Report: results from the 2014 Scottish Health Survey. Edinburgh: Scottish Government; 2015.
4. Heaney CA, Israel BA. Social networks and social support. In: Glanz K, Rimer BK, Viswanath K, editors. Health behavior and health education: theory, research, and practice. San Francisco: Wiley; 2008. p. 189-210.

5. Karfopoulou E, Anastasiou CA, Avgeraki E, Kosmidis MH, Yannakoulia M. The role of social support in weight loss maintenance: results from the MedWeight study. J Behav Med. 2016;39(3):511-8.

6. Greaves CJ, Sheppard KE, Abraham C, Hardeman W, Roden M, Evans PH, et al. Systematic review of reviews of intervention components associated with increased effectiveness in dietary and physical activity interventions. BMC Public Health 2011;11(1):1-12.

7. Baird J, Cooper C, Margetts B, Barker M, Inskip H. Changing health behaviour of young women from disadvantaged backgrounds: evidence from systematic reviews. P Nutr Soc. 2009;68(02):195-204

8. Simpson SA, Shaw C, McNamara R. What is the most effective way to maintain weight loss in adults. BMJ. 2011;343:d8042

9. Michie S, Abraham C, Whittington C, McAteer J, Gupta S. Effective techniques in healthy eating and physical activity interventions: a metaregression. Health Psychol. 2009;28(6):690-701.

10. Stewart DW, Gabriele JM, Fisher EB. Directive support, nondirective support, and health behaviors in a community sample. J Behav Med. 2012;35(5):492-9.

11. Bandura A. Health promotion from the perspective of social cognitive theory. Psychol Health. 1998;13(4):623-49.

12. Carver CS, Scheier MF. Control theory: a useful conceptual framework for personality-social, clinical, and health psychology. Psychol Bull. 1982;92(1):111-35.

13. Wei J, Hollin I, Kachnowski S. A review of the use of mobile phone text messaging in clinical and healthy behaviour interventions. J Telemed Telecare. 2011;17(1):41-8.

14. Free C, Knight R, Robertson S, Whittaker R, Edwards P, Zhou W, et al. Smoking cessation support delivered via mobile phone text messaging (txt2stop): a single-blind, randomised trial. Lancet. 2011;378:49-55.

15. Simpson SA, et al. An app-, web- and social support-based weight loss intervention for adults with obesity: the HelpMeDolt! feasibility RCT. Public Health Res. 2020;8(3). https://doi.org/10.3310/phr08030.

16. Craig P, Dieppe P, Macintyre S, Michie S, Nazareth I, Petticrew M. Developing and evaluating complex interventions: the new Medical Research Council guidance. BMJ. 2008;337:a1655.

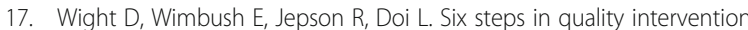
development (6SQuID). J Epidemiol Community Health. 2015.

18. Yardley L, Williams S, Bradbury K, Garip G, Renouf S, Ware L, et al. Integrating user perspectives into the development of a web-based weight management intervention. Clinical Obesity. 2012;2:132-41.

19. Matthews L, Pugmire J, Moore L, Kelson M, McConnachie A, Mclntosh E, et al. Study protocol for the 'HelpMeDolt!' randomised controlled feasibility trial: an app, web and social support-based weight loss intervention for adults with obesity. BMJ Open. 2017;7(10):e017159.

20. Lund AM. Measuring usability with the USE Questionnaire. STC Usability SIG newsletter. 2001;8(2).

21. Roe L, Strong C, Whiteside C, Neil A, Mant D. Dietary intervention in primary care: validity of the DINE method for diet assessment. Fam Pract. 1994;11(4):375-81.

22. Freedson PS, Melanson E, Sirard J. Calibration of the Computer Science and Applications Inc. accelerometer. Med Sci Sports Exerc. 1998;30:777-81.

23. Hayden-Wade HA, Coleman KJ, Sallis JF, Armstrong C. Validation of the telephone and in-person interview versions of the 7-day PAR. Med Sci Sports Exerc. 2003;35(5):801-9.

24. EuroQol Group. EuroQol - a new facility for the measurement of health related quality of life. Health Policy. 1990;16:199-208.

25. Al-Janabi H, Flynn T. Development of a self-report measure of capability wellbeing for adults: the ICECAP-A. Qual Life Res. 2012;21:167-76.

26. Goldberg DP, Williams P. A user's guide to the General Health Questionnaire. Basingstoke: NFER Nelson; 1988.

27. Sallis JF, Grossman RM, Pinski RB, Patterson TL, Nader PR. The development of scales to measure social support for diet and exercise behaviors. Prev Med. 1987;16(6):825-36.

28. Clark MM, Abrams DB, Niaura RS, Eaton CA, Rossi JS. Self-efficacy in weight management. J Consult Clin Psychol. 1991;59(5):739-44.

29. Rodgers WM, Wilson PM, Hall CR, Fraser SN, Murray TC. Evidence for a multidimensional self-efficacy for exercise scale. Res Q Exerc Sport. 2008; 79(2):222-34.

30. Rodgers WM, Sullivan MJL. Task, coping, and scheduling self-efficacy in relation to frequency of physical activity. J Appl Soc Psychol. 2001;31(4):741-53. 
31. Levesque CS, Williams GC, Elliot D, Pickering MA, Bodenhamer B, Finley PJ. Validating the theoretical structure of the Treatment Self-Regulation Questionnaire (TSRQ) across three different health behaviors. Health Educ Res. 2007;22(5):691-702.

32. Heatherton TF, Kozlowski LT, Frecker RC, Rickert W, Robinson J. Measuring the heaviness of smoking -using self reported time to the first cigarette of the day and number of cigarettes smoked per day. Br J Addict. 1989;84(7):791-800.

33. Bradley KA, DeBenedetti AF, Volk RJ, Williams EC, Frank D, Kivlahan DR. AUDIT-C as a brief screen for alcohol misuse in primary care. Alcohol Clin Exp Res. 2007;31(7):1208-17.

34. Bingham SA. Limitations of the various methods for collecting dietary intake data. Ann Nutr Metab. 1991;35(3):117-27.

35. Prince S, Adamo K, Hamel M, Hardt J, Gorber S, Tremblay M. A comparison of direct versus self-report measures for assessing physical activity in adults: a systematic review. IJBNPA. 2008;5(56):1-24.

36. Holmes B, Dick K, Nelson M. A comparison of four dietary assessment methods in materially deprived households in England. Public Health Nutr. 2008;11(5):444-56.

37. Sallis JF, Haskell WL, Wood PD, Fortmann SP, Rogers T, Blair SN, et al. Physical activity assessment methodology in the Five-City Project. Am J Epidemiol. 1985;121(1):91-106.

38. Curtis L. Unit costs of health and social care 2013: Personal Social Services Research Unit (PSSRU); 2013.

39. Eldridge SM, Chan CL, Campbell MJ, Bond CM, Hopewell S, Thabane L, et al. CONSORT 2010 statement: extension to randomised pilot and feasibility trials. BMJ Open. 2016;355(i5239).

40. Braun V, Clarke V. Using thematic analysis in psychology. Qual Res Psychol. 2006;3(2).

41. Qualitative Research in Health Care. 3rd ed. Massachusettes, USA: Blackwell Publishing Ltd; July 1, 2006.

42. Warner E, Wolin K, Duncan D, Heil D, Askew S, Bennett G. Differential accuracy of physical activity self-report by body mass index. Am J Health Behav. 2012;36(2):168-78

43. Localytics. $21 \%$ of users abandon an app after first use 2018 [01/05/18]. Available from: http://info.localytics.com/blog/21-percent-of-users-abandonapps-after-one-use.

44. Laing BY, Mangione CM, Tseng $\mathrm{CH}$, Leng M, Vaisberg $\mathrm{E}$, Mahida $\mathrm{M}$, et al. Effectiveness of a smartphone application for weight loss compared with usual care in overweight primary care patients: a randomized, controlled trial. Ann Intern Med. 2014;161(10 Suppl):S5-12.

45. Carter MC, Burley VJ, Nykjaer C, Cade JE. Adherence to a smartphone application for weight loss compared to website and paper diary: pilot randomized controlled trial. J Med Internet Res. 2013;15(4):e32.

46. Michie S, Yardley L, West R, Patrick K, Greaves F. Developing and evaluating digital interventions to promote behavior change in health and health care: recommendations resulting from an international workshop. J Med Internet Res. 2017;19(6):e232.

47. Ford JG, Howerton MW, Lai GY, Gary TL, Bolen S, Gibbons MC, et al. Barriers to recruiting underrepresented populations to cancer clinical trials: a systematic review. Cancer. 2008;112:228-42.

48. White M, Adams J, Heywood P. How and why do interventions that increase health overall widen inequalities within populations? In: SJ. B, editor. Social inequality and public health. Bristol: Policy Press; 2009. p. 64-81.

49. Sharpe PA, Wilcox S, Rooney L, Strong D, Hopkins-Campbell R, Butel J, et al. Adherence to accelerometer protocols among women from economically disadvantaged neighborhoods. J Phys Act Health. 2011;8(5):699-706.

50. Audrey S, Bell S, Hughes R, Campbell R. Adolescent perspectives on wearing accelerometers to measure physical activity in population-based trials. Eur $J$ Pub Health. 2012;23(3):475-80.

\section{Publisher's Note}

Springer Nature remains neutral with regard to jurisdictional claims in published maps and institutional affiliations.

Ready to submit your research? Choose BMC and benefit from:
- fast, convenient online submission
- thorough peer review by experienced researchers in your field
- rapid publication on acceptance
- support for research data, including large and complex data types
- gold Open Access which fosters wider collaboration and increased citations
- maximum visibility for your research: over 100M website views per year
At BMC, research is always in progress.
Learn more biomedcentral.com/submissions

